OUTP-02/44P

HIP-2002-65/TH

hep-th/0212137

December 2002

\title{
GHOST-MATTER MIXING AND FEIGENBAUM UNIVERSALITY IN STRING THEORY
}

\author{
Ian I. Kogan ${ }^{1 a, b}$ and Dimitri Polyakov ${ }^{2 c}$ \\ a Theoretical Physics, Department of Physics, Oxford University, 1 Keble Road, Oxford, \\ $O X 13 N P, U K$ \\ ${ }^{b}$ ITEP, Moscow, Russia \\ ${ }^{c}$ Department of Physical Sciences, University of Helsinki and Helsinki Institute of \\ Physics, PL 64, FIN-00014 Helsinki, Finland
}

Dedicated to the 80-th birthday of Karen Avetovich Ter-Martirosyan.

\begin{abstract}
Brane-like vertex operators, defining backgrounds with the ghost-matter mixing in Neveu-Schwarz-Ramond (NSR) superstring theory, play an important role in a world-sheet formulation of D-branes and M theory, being creation operators for extended objects in the second quantized formalism. In this paper we show that dilaton's beta function in ghostmatter mixing backgrounds becomes stochastic. The renormalization group (RG) equations in ghost-matter mixing backgrounds lead to non-Markovian Fokker-Planck equations which solutions describe superstrings in curved space-times with brane-like metrics. We show that Feigenbaum universality constant $\delta=4,669 \ldots$ describing transitions from order to chaos in a huge variety of dynamical systems, appears analytically in these RG equations. We find that the appearance of this constant is related to the scaling of relative space-time curvatures at fixed points of the RG flow. In this picture the fixed points correspond to the period doubling of Feigenbaum iterational schemes.
\end{abstract}

\footnotetext{
1i.kogan@physics.ox.ac.uk

22polyakov@pcu.helsinki.fi
} 


\section{Introduction}

Superstring theory is our current hope to put gravity in a Prokrust's bed of quantum mechanics. In spite of all the spectacular progress in the last quarter of the century [1] the full structure and underlying symmetries of the theory have yet to be unveiled. One of the most striking features of String theory is a deep relation between renormalization group (RG) flows on a world sheet and an evolution in a target space. Critical points of these RG flows, described by $2 \mathrm{~d}$ conformal field theories (CFT), determine equations of motion in a target space. The structure of these equations is determined by the world sheet correlation functions of the appropriate vertex operators in respective CFT [2] Thus in the standard string perturbation theory the beta beta-function equations describe the behaviour of small fluctuations around flat backgrounds. The conformal field theory description of strings in curved backgrounds, such as of strings in the presence of branes, as well as the underlying CFT of strongly coupled strings is much harder a problem to tackle, in particular because the adequate knowledge of quantum degrees of freedom of M-theory and non-perturbative strings is still lacking. Some time ago, we have proposed the formalism [3]-[6] that describes the non-perturbative dynamics of solitons in string and M-theory in terms of a special class of vertex operators, called brane-like states. The crucial distinction of these vertex operators from usual one (such as a photon or a graviton) is that they exist at nonzero ghost pictures only. The simplest example of these vertices in the closed string case is given by:

$$
\begin{array}{r}
V_{5}^{(-3)}(q)=\int d^{2} z e^{-3 \phi-\bar{\phi}} \psi_{t_{1}} \ldots \psi_{t_{5}} \bar{\psi}_{t_{6}} e^{i q_{a} X_{a}}(z, \bar{z}) \\
V_{5}^{(-2)}(q)=\int d^{2} z c \partial \chi e^{\chi-3 \phi-\bar{\phi}} \psi_{t_{1}} \ldots \psi_{t_{5}} \bar{\psi}_{t_{6}} e^{i q_{a} X_{a}}(z, \bar{z}) \\
V_{5}^{(+1)}(q)=\int d^{2} z e^{\phi-\bar{\phi}} \psi_{t_{1}} \ldots \psi_{t_{5}} \bar{\psi}_{t_{6}} e^{i q_{a} X_{a}}(z, \bar{z})+b-c \text { ghosts } \\
a=0, \ldots, 3 ; \quad t_{i}=4, \ldots 9 ;
\end{array}
$$

It is important that the BRST nontriviality and invariance conditions on this vertex confine its propagation to the 4-dimensional subspace (labeled by the $a$ index), transverse to its polarization defined by $6 t_{i}$ indices. This six-form vertex exists only at pictures below -2 or above +1 while there is no version of this operator at pictures 0 and -1 .

This means that the discrete picture-changing gauge symmetry is broken for such operators and their superconformal ghost dependence cannot be removed by any picture-changing transformation. We shall refer to this property of the brane-like vertices as the ghost-matter 
mixing. The crucial property of these special vertex operators is that they do not correspond to any perturbative string excitation but describe the nonperturbative dynamics of extended solitonic objects, such as D-branes. It appears that the non-perturbative character of these vertices is closely related to the their ghost-matter mixing properties on the world sheet, with the latter encoding the crucial information about the brane dynamics. In [6] we have shown that the low-energy effective action of the sigma-model with the brane-like states is given by the Dirac-Born-Infeld (DBI) action for D-branes. From the world sheet point of view this means that the insertion of vertices with the ghost-matter mixing makes the deform CFT describing strings in flat space-time and it flows to a new fixed point, corresponding to the CFT of strings in a curved background induced by D-branes. In this paper we shall further investigate RG flows in the ghost-matter mixing backgrounds. It appears that properties of these RG flows are stunningly different from the usual ones. We found that ghost-matter mixing adds to RG flow operator-valued stochastic terms. Even more intriguing is the emergence of universal constant in the RG equations which with accuracy less than $0.5 \%$ is nothing but the logarithm of the famous Feigenbaum constant $\delta=4.669$ [7]. This coincidence is not accidental but reflects remarkable and new relations between superstrings, chaos, gravity and stochastic processes which is the subject of this Letter.

\section{Dilaton beta-function in ghost-matter mixing back- grounds}

The crucial property of world sheet conformal beta-functions (e.g. of a dilaton) in ghostmatter mixing backgrounds is the presence of stochastic terms in the RG equations. In usual perturbative backgrounds (e.g. of a graviton or an axion) such terms are absent and the beta-function equations are deterministic. Things, however, become different in backgrounds with the ghost-matter mixing. One specific property of the brane-like states, distinguishing them from usual perturbative vertex operators, is that their OPE algebra is picture-dependent. Below we will show that this picture dependence leads to non-deterministic stochastic terms in the dilaton's beta-function. The stochasticity of the renormalization group, in turn, will be shown to be closely related to the nonperturbative nature of the brane-like states and their relevance to the non-perturbative description of branes and strings in curved backgrounds. Let us start with the Neveu-Schwarz-Ramond (NSR) sigma model in $D=10$ perturbed by the dilaton and the ghost-matter mixing vertex 
(1). The generating functional for this model is given by:

$$
\begin{array}{r}
Z(\varphi, \lambda)=\int D X D \psi D[\text { ghosts }]: f(\Gamma):: f(\bar{\Gamma}): \\
\exp \left\{-S_{N S R}+\int d^{4} q \lambda(q) \int d^{2} z V_{5}^{(-3)}(q, z, \bar{z})+\int d^{10} p \varphi(p) \int d^{2} w V_{\varphi}^{(-2)}(p, w, \bar{w})\right\}
\end{array}
$$

Here $V_{5}^{(-3)}$ is the ghost-matter mixing vertex $(1)$, creating the D3-brane background, $V_{\varphi}^{(-2)}$ is the dilaton vertex operator taken at the ghost picture -2 .

$$
: f(\Gamma):=: \frac{1}{1-\Gamma}:=1+: \Gamma:+: \Gamma^{2}:+\ldots
$$

is the measure function of picture-changing operator, : $\Gamma:=: e^{\phi} G$ : with $G=G_{m}+G_{g h}$ being the full matter + ghost world sheet supercurrent. This measure function is necessary to insure the correct ghost number balance in correlation function; : $f(\bar{\Gamma})$ : is defined similarly. In the perturbative case the : $f(\Gamma) f(\bar{\Gamma})$ : insertion simply insures that all the operator product expansions (OPE) of the vertex operators are equivalent at all the picture levels and all the structure correlation functions are picture independent. The dilaton vertex operator can be taken at any negative picture. The negative value of the dilaton picture, along with the : $f(\Gamma)$ : in the measure, insures the correct overall ghost number in correlation functions involving the dilaton (which must be equal to -2 on the sphere) this vertex operator is picture independent (meaning the picture independence its matrix elements with other states) and the results of the dilaton's beta-function are independent on the $V_{\varphi}$ 's picture as well. It is convenient to take $V_{\varphi}$ at picture -2 (both left and right) as in this case the dilaton vertex operator is given by

$$
V_{\varphi}(p)=\int d^{2} z e^{-2 \phi-2 \bar{\phi}} \partial X^{m} \partial X_{n}\left(\eta_{m n}-k_{m} \bar{k}_{n}-k_{n} \bar{k}_{m}\right)
$$

- In the absence of ghost-matter mixing the functions : $f(\Gamma):$ and $: f(\bar{\Gamma})$ : simply insure that the beta-function equations for any closed string space-time fields are identical for any picture representation of corresponding closed string vertex operators. In order to compute the dilaton's beta-function in the presence of the $V_{5}$-operator, one has to expand this generating functional in $\varphi$ and $\lambda$. In the absence of ghost-matter mixing, the dilaton's (or any other closed string field's) beta-function in any background is simply given by the appropriate three-point correlators, or the structure constants. In the ghost-matter 
mixing case, however, the situation is different since the structure constants are picturedependent and therefore expressing the beta-function in terms of three-point correlation functions is not well-defined. Therefore to determine the beta-function one has to point out the UV logarithmic divergences due to the OPE singularities (generally speaking, picture dependent) and to sum over all the pictures.

Expanding the generating functional (2) up to the third order of $\lambda$ and the second order of $\varphi$ we obtain:

$$
\begin{array}{r}
Z(\varphi, \lambda)=\int D X D \psi D[\text { ghosts }]: f(\Gamma):: f(\bar{\Gamma}): e^{-S_{N S R}} \\
\left\{1+\int d^{10} p \varphi(p) \int d^{2} z V_{\varphi}^{(-2)}(z, \bar{z} ; p)+\int d^{4} q \lambda(q) \int d^{2} w V_{5}^{(-3)}(w, \bar{w} ; q)\right. \\
+\int d^{10} p \int d^{4} q \lambda(q) \varphi(p) \int d^{2} z \int d^{2} w V_{\varphi}^{(-2)}(p ; z, \bar{z}) V_{5}^{(-3)}(w, \bar{w} ; q) \\
+\frac{1}{2} \int d^{4} q_{1} \lambda\left(q_{1}\right) \int d^{4} q_{2} \lambda\left(q_{2}\right) \int d^{2} w_{1} \int d^{2} w_{2} V_{5}^{(-3)}\left(w_{1}, \bar{w}_{1} ; q_{1}\right) V_{5}^{(-3)}\left(w_{2}, \bar{w}_{2} ; q_{2}\right) \\
+\frac{1}{2} \int d^{10} p_{1} \varphi\left(p_{1}\right) \int d^{10} p_{2} \varphi\left(p_{2}\right) \int d^{2} z_{1} \int d^{2} z_{2} V_{\varphi}^{(-2)}\left(z_{1}, \bar{z}_{1} ; p_{1}\right) V_{\varphi}^{(-2)}\left(z_{2}, \overline{z_{2}} ; p_{2}\right) \\
+\frac{1}{6} \int d^{4} q_{1} \int d^{4} q_{2} \int d^{4} q_{3} \lambda\left(q_{1}\right) \lambda\left(q_{2}\right) \lambda\left(q_{3}\right) \\
\left.\times \int d^{2} w_{1} \int d^{2} w_{2} \int d^{2} w_{3} V_{5}^{(-3)}\left(w_{1}, \bar{w}_{1} ; q_{1}\right) V_{5}^{(-3)}\left(w_{2}, \bar{w}_{2} ; q_{2}\right) V_{5}^{(-3)}\left(w_{3}, \bar{w}_{3} ; q_{3}\right)+\ldots\right\}
\end{array}
$$

where we dropped the higher order terms as well as those irrelevant to our discussion (such as those of the order of $\lambda^{2} \varphi$ or $\varphi^{2} \lambda$ ). To determine the UV divergences in the partition function (2),(3), relevant to the dilaton's beta-function, one has to point out the relevant singular terms in the OPE algebra of the dilaton and $V_{5}$. In the on-shell limit, the relevant terms in the operator algebra are given by:

$$
\begin{aligned}
V_{5}^{(-3)}\left(w_{1}, \bar{w}_{1} ; q_{1}\right) V_{5}^{(-3)}\left(w_{2}, \bar{w}_{2} ; q_{2}\right) & \sim \frac{C_{[-3 \mid-3]}\left(q_{1}, q_{2}\right) V_{\varphi}^{(-6)}\left(q_{1}+q_{2}\right)}{\left|w_{1}-w_{2}\right|^{2}}+\ldots \\
V_{5}^{(-3)}\left(w_{1}, \bar{w}_{1} ; q_{1}\right) V_{5}^{(+1)}\left(w_{2}, \bar{w}_{2} ; q_{2}\right) & \sim \frac{C_{[-3 \mid 1]}\left(q_{1}, q_{2}\right) V_{\varphi}^{(-2)}\left(q_{1}+q_{2}\right)}{\left|w_{1}-w_{2}\right|^{2}}+\ldots \\
V_{5}^{(+1)}\left(w_{1}, \bar{w}_{1} ; q_{1}\right) V_{5}^{(+1)}\left(w_{2}, \bar{w}_{2} ; q_{2}\right) & \sim \frac{C_{[1 \mid 1]}\left(q_{1}, q_{2}\right) V_{\varphi}^{(+2)}\left(q_{1}+q_{2}\right)}{\left|w_{1}-w_{2}\right|^{2}}+\ldots
\end{aligned}
$$

where

$$
C_{[-3 \mid-3]}\left(q_{1}, q_{2}\right) \sim\left(q_{1} q_{2}\right)\left(1+\left(q_{1}+q_{2}\right)^{2}\right)
$$




$$
\begin{array}{r}
C_{[-3 \mid 1]}\left(q_{1}, q_{2}\right) \sim\left(q_{1} q_{2}\right) \\
C_{[-3 \mid 1]}\left(q_{1}, q_{2}\right) \sim\left(q_{1} q_{2}\right)\left(1-\left(q_{1}+q_{2}\right)^{2}\right)
\end{array}
$$

Next, one has to point out the picture changing rules for the left (holomorphic) part of the $V_{5}$-operator, in order to specify how it is acted on by $: f(\Gamma)$ : The antiholomorphic part and its interaction with : $f(\bar{\Gamma})$ : do not interest us since it is the photon-like part existing at all pictures and all its picture changings are trivial. The picture changing transformation rules for the $V_{5}$ operators (1) can be written in the form

$$
\begin{gathered}
: \Gamma:^{n} V_{5}^{(k)}(p)=\alpha_{[k \mid n+k]} V_{5}^{(N+k)}(p) \\
\alpha_{[i \mid j]}=\alpha_{[m \mid n]}=\alpha_{[s \mid t]}=1 ; \quad \alpha_{[a \mid j]}=\alpha_{[a \mid b]}=\alpha_{[s \mid a]}=0, \quad \alpha_{[i \mid m]}=\alpha_{[s \mid m]}=1+p^{2}, \\
s, t=-\infty, \ldots-4 ; \quad i, j=-3,-2 ; \quad a, b=-1,0 ; \quad m, n=1,2, \ldots .
\end{gathered}
$$

In the beta-function calculations, when the vertex operators are taken just slightly off-shell, the following identities are useful:

$$
\alpha_{[i \mid m]} C_{[m \mid n]}=C_{[i \mid n]} ; \quad \alpha_{[i \mid m]} C_{[m \mid j]}=C_{[i \mid j]}
$$

Finally, using the fact that picture changing operators form the polynomial ring:

$$
: \Gamma:^{m+n}=: \Gamma^{m} \Gamma^{n}:+\left[Q_{B R S T}, \ldots\right]
$$

the action of the : $\Gamma^{n}$ : operator on the vertex operators inside the functional integral can be expressed as:

$$
\begin{array}{r}
<: \Gamma^{n}:(w) V_{1}\left(z_{1}\right) \ldots . V_{N}\left(z_{N}\right)>=\sum_{k_{1}, \ldots k_{N-1}=0}^{k_{1}+\ldots+k_{N-1}=n} N^{-n} \frac{n !}{k_{1} ! \ldots k_{N-1} !\left(n-k_{1}-\ldots-k_{N-1}\right) !} \\
\quad \times<: \Gamma:^{k_{1}} V_{1}\left(z_{1}\right) \ldots: \Gamma:^{k_{N-1}} V_{N-1}\left(z_{N-1}\right): \Gamma:^{n-k_{1}-\ldots-k_{N}} V_{N}\left(z_{N}\right)>
\end{array}
$$

i.e. the correlator does not depend on $w$. The factor of $N^{-n}$ in (9) insures the correct normalization of amplitudes in the picture-independent case. Using the relations (6)-(9) we are finally in the position to start evaluating the beta-function. The first contribution of interest to the beta-function comes from the $\lambda^{2}$-term, bilinear in the $V_{5}$-operator. At any given picture level $n$ this term leads to the following divergence in the order of $\lambda^{2}$ :

$$
\begin{array}{r}
\frac{1}{2} \int d^{2} w_{1} \int d^{2} w_{2}<: \Gamma^{n+6}: V_{5}^{(-3)}\left(w_{1}, \bar{w}_{1}\right) V_{5}^{(-3)}\left(w_{2}, \bar{w}_{2}\right) \ldots>= \\
=\log \Lambda \times 2^{-n-7} \sum_{k=0}^{n+6} \frac{(n+6) !}{k !(n+6-k) !} \alpha_{[-3 \mid k-3]} C_{[k-3 \mid n+3-k]} \alpha_{[-3 \mid n+3-k]} \int d^{2} \xi<V_{\varphi}^{(n)}(\xi, \bar{\xi}) \ldots>(10
\end{array}
$$


where we introduced the coordinate change: $\xi=1 / 2\left(w_{1}+w_{2}\right), \eta=1 / 2\left(w_{1}-w_{2}\right)$ and $\log \Lambda=$ $\int_{\Lambda} \frac{d^{2} \eta}{|\eta|^{2}}$ is the log of the world sheet UV cutoff, appearing as a result of the integration over $\eta$ (note that the vertex operators on the r.h.s. of the operator product (10) are $\eta$-independent) For the sake of brevity we suppress the momentum dependence of fields, vertices and structure constants here and below. This divergence is removed by renormalizing the dilaton field as

$$
\varphi \rightarrow \varphi-\sum_{n=0}^{\infty} 2^{-n-7} \sum_{k=0}^{n+6} \frac{(n+6) !}{k !(n+6-k) !} \alpha_{[-3 \mid k-3]} C_{[k-3 \mid n+3-k]} \alpha_{[-3 \mid n+3-k]} \lambda^{2} \log \Lambda
$$

In the absence of picture-dependence the sum over $\mathrm{k}$ would have been reduced to $\frac{1}{2} C \lambda^{2} \log \Lambda$ for each picture, as it should be in the standard case when ghost-matter mixing is absent (where $\mathrm{C}$ are the structure constants with the picture indices suppressed)

As a result of the dilaton's RG flow, the $\lambda \varphi$ cross-term is renormalized by $\lambda^{3}$ logarithmic divergence as:

$$
\begin{array}{r}
\lambda \varphi \int d^{2} w_{1} \int d^{2} w_{2}<: f(\Gamma): V_{5}^{(-3)} V_{\varphi}^{(-2)} . .> \\
\lambda^{3} \log \Lambda \sum_{n=0}^{\infty} 2^{-2 n-12} \sum_{k, l=0}^{k+l=n+5} \frac{(n+5) !(n+6) !}{k ! l !(n+5-l) !(n+6-k) !} \alpha_{[-3 \mid n+2-l]} \alpha_{[-3 \mid k-3]} \\
\times \alpha_{[-3 \mid n+3-k]} C_{[k-3 \mid n+3-k]} \int d^{2} w_{1} \int d^{2} w_{2}<V_{\varphi}^{(-6)}\left(w_{1}, \bar{w}_{1}\right) V_{5}^{(n+6)}\left(w_{2}, \bar{w}_{2}\right) \ldots>
\end{array}
$$

where in our derivation we used the invariance of $V_{\varphi}$ and transformation properties (6) of $V_{5}$ under the picture-changing. In the absence of the ghost-matter mixing when one always has $\alpha=1$ and picture-independent $C$ it is easy to check that the $\lambda \varphi$ would have been renormalized to

$$
-\frac{1}{2} C \lambda^{3} \log \Lambda \int d^{2} w_{1} \int d^{2} w_{2} V\left(w_{1}, \bar{w}_{1}\right) V\left(w_{2}, \bar{w}_{2}\right)
$$

at each picture level (again, we suppressed the relevant indices for the structure constants and vertex operators) In the case under consideration, however, using the identities (7) relating $\alpha$ and $C$, we can cast the renormalization of the $\lambda \varphi$-term under the flow (11) as

$$
\begin{array}{r}
A_{\lambda \varphi} \sim \lambda \varphi: f(\Gamma): \lambda \varphi \int d^{2} w_{1} \int d^{2} w_{2} V_{5}^{(-3)} V_{\varphi}^{(-2)} \rightarrow \\
-\lambda^{3} \log \Lambda C_{[-3 \mid-3]} \alpha_{[-3 \mid 1]} \int d^{2} w_{1} \int d^{2} w_{2} V_{\varphi}^{(-6)}\left(w_{1}, \bar{w}_{1}\right) V_{5}^{(n+6)}\left(w_{2}, \bar{w}_{2}\right)
\end{array}
$$




$$
\times \sum_{n=0}^{\infty} \sum_{k, l=0 ; k \neq 2,3, n+3, n+4 ; l \neq n+2, n+3}^{k=n+6 ; l=n+5} \frac{(n+5) !(n+6) !}{k ! l !(n+5-l) !(n+6-k) !}
$$

This gives the renormalization of the $\lambda \varphi$ cross-term under the RG flow (11) of the dilaton field in the ghost-matter mixing case. This contribution to the world sheet renormalization group has the order of $\lambda^{3}$ The other contribution of the same order of $\lambda^{3}$ to the dilaton beta-function comes from the OPE singularities inside the $\lambda^{3}$-term itself, appearing in the expansion (3) of the partition function Using the OPE (4) and evaluating the singular world sheet integrals as in (10), (11) we get

$$
\begin{aligned}
& A_{\lambda^{3}}=\frac{1}{6} \lambda^{3} \int d^{2} w_{1} \int d^{2} w_{2} \int d^{2} w_{3}<V_{5}^{(-3)}\left(w_{1}, \bar{w}_{1}\right) V_{5}^{(-3)}\left(w_{2}, \bar{w}_{2}\right) V_{5}^{(-3)}\left(w_{3}, \bar{w}_{3}\right) \ldots> \\
& \sim \frac{1}{2} \lambda^{3} \log \Lambda \sum_{n=0}^{\infty} 3^{-n-10} \sum_{k, l=0}^{k+l=n+9} \frac{(n+9) !}{k ! l !(n+9-k-l) !} \\
& \times \int d^{2} w_{1} \int d^{2} w_{2}<V_{\varphi}^{(-6)}\left(w_{1}, \bar{w}_{1}\right) V_{5}^{(n+6)}\left(w_{2}, \bar{w}_{2}\right) \ldots> \\
&\left\{C_{[k-3 \mid l-3]} \alpha_{[-3 \mid k-3]} \alpha_{[-3 \mid n+6-k-l]} \alpha_{[-3 \mid n+6]}+C_{[k-3 \mid n+6-k-l]} \alpha_{[-3 \mid k-3]} \alpha_{[-3 \mid l-3]} \alpha_{[-3 \mid n+6]}\right. \\
&\left.+C_{[k-3 \mid n+6-k-l]} \alpha_{[-3 \mid l-3]} \alpha_{[-3 \mid n+6-k-l]} \alpha_{[-3 \mid n+6]}\right\}
\end{aligned}
$$

Again, it is easy to see that in the absence of the ghost-matter mixing $(\alpha=1$, all $\mathrm{C}$ are picture-independent) this contribution would sum up to

$$
\frac{1}{2} C \lambda^{3} \log \Lambda \int d^{2} w_{1} \int d^{2} w_{2} V\left(w_{1}, \bar{w}_{1}\right) V\left(w_{2}, \bar{w}_{2}\right)
$$

precisely cancelling the divergence of the same $\lambda^{3}$-type, originating from the renormalization of the $\lambda \varphi$ cross term under the flow. In the picture-independent case this insures that the renormalization (11) of the dilaton field under the flow does not bring about any additional singularities from higher order terms, such as the cubic one and the $\lambda \varphi$ cross-term. In particular, this guarantees that terms of the type

$$
\sim C \lambda^{3} \log \Lambda \int_{\Lambda} d^{2} w V_{5}(w, \bar{w})
$$

( $\int_{\Lambda}$ denotes the world sheet integral cut at the $\Lambda$ scale) never appear in the dilaton or other perturbative close string field beta-functions in the picture-independent case. This insures, in turn, that in the absence of the ghost-matter mixing the world sheet betafunction is always deterministic (just as it is well-known to be the case in the standard 
string perturbation theory) On the contrary, should the terms of this type appear in the beta-function, that would imply that the RG equations become stochastic, since from the point of view of the space-time fields, world sheet operator $\int_{\Lambda} d^{2} w V_{5}(w, \bar{w})$ is a stochastic random variable, with the cutoff parameter $\Lambda$ playing the role of the stochastic time. In this case, the $\mathrm{RG}$ equations have the form of non-Markovian Langevin equations where the memory of the noise is determined by the world sheet correlation of the $V_{5}$-operators. This exactly is what happens in the ghost-matter mixing backgrounds, when the OPE of vertex operators are picture-dependent. As a result of the picture-dependence, the flows of the $\lambda \varphi$ and $\lambda^{3}$ terms under the RG do not cancel each other and, as a result, the beta-function of the dilaton gets the stochastic terms, as $A_{\lambda^{3}}+A_{\lambda \varphi} \neq 0$ Indeed, using the identities (7) we can write (15) in the form:

$$
\begin{array}{r}
\frac{1}{2} C_{[-3 \mid-3]} \alpha_{[-3 \mid 1]} \lambda^{3} \log \Lambda \sum_{n=0}^{\infty} 3^{-n-9} \sum_{k, l=0 ; k \neq 2,3 ; l \neq 2,3 ; k+l \neq n+5, n+6}^{k+l=n+9} \frac{(n+9) !}{k ! l !(n+9-k-l) !} \\
\int d^{2} w_{1} \int d^{2} w_{2}<V_{\varphi}^{(-6)}\left(w_{1}, \bar{w}_{1}\right) V_{5}^{(n+6)}\left(w_{2}, \bar{w}_{2}\right) \ldots>
\end{array}
$$

giving the contribution of the $A_{\lambda^{3}}$-term to the flow. Now, to get the total flow on the $\lambda^{3}$ level one has to subtract the flow (14) from this expression. The difference would give the overall coefficient before the stochastic term of the type (17) in the beta-function of the dilaton. Comparing (14) and (18) and performing the summations we find that the additional renormalization of the dilaton field, necessary to remove the extra singularities from the $\lambda^{3}$ and $\lambda \varphi$ terms, arising due to the OPE picture dependence in ghost-matter mixing backgrounds, is given by

$$
\begin{array}{r}
\varphi \rightarrow \varphi-C_{[-3 \mid-3]} \alpha_{[-3 \mid 1]} \lambda^{3} \log \Lambda \int d^{2} w V_{5}^{(-3)}(w, \bar{w}) \\
\times\left\{-1+\sum_{n=0}^{\infty}\left[(n+4)^{2}(n+5)^{3}(n+6) 2^{-2 n-12}+\frac{1}{96}(n+8)(n+9)(n+13)\left(\frac{2}{3}\right)^{n+9}-\right.\right. \\
\left.\left.\left((n+4)^{2}(n+5)+(1 / 2)(n+5)^{2}(n+6)\right) 2^{-n-6}\right]\right\}
\end{array}
$$

The summation over $\mathrm{n}$ converges to

$$
\begin{array}{r}
-\sigma=-1+\sum_{n=0}^{\infty}\left[(n+4)^{2}(n+5)^{3}(n+6) 2^{-2 n-12}+\frac{1}{96}(n+8)(n+9)(n+13)\left(\frac{2}{3}\right)^{n+9}\right. \\
\left.-\left((n+4)^{2}(n+5)+(1 / 2)(n+5)^{2}(n+6)\right) 2^{-n-6}\right]=-1,534 .
\end{array}
$$


Therefore the resulting beta-function equations for the dilaton in the ghost-matter mixing background give:

$$
\frac{d \varphi}{d \log \Lambda}=-\frac{\delta S_{\varphi}}{\delta \varphi}+\sigma C_{[-3 \mid-3]} \alpha_{[-3 \mid 1]} \int_{\Lambda} d^{2} w V_{5}^{(-3)}(w, \bar{w})
$$

where $S_{\varphi} \sim \int d x \partial_{m} \varphi \partial^{m} \varphi$ is the low energy effective action for the dilaton in the absence of the $V_{5}$ background.

With the restored momentum dependence, this equation can be written as

$$
\begin{array}{r}
\frac{d \varphi(p)}{d \log \Lambda}=-\frac{\delta S_{\varphi}}{\delta \varphi(p)}+\sigma C(p) \int d^{4} q \lambda(q) \int_{\Lambda} d^{2} w V_{5}^{(-3)}(w, q) \\
C(p)=\int d^{4} k C_{[-3 \mid-3]}(p, k) \lambda\left(\frac{k+p}{2}\right) \lambda\left(\frac{k-p}{2}\right)
\end{array}
$$

with $\sigma=1,534 \ldots$ So far we were considering only one particular example of the ghost-matter mixing - the brane-like vertex operator $V_{5}^{(-6)}$ and its incarnations in higher pictures. There are other examples of vertex operators with ghost-matter mixing, and they also lead to stochastic terms in the beta-function of the dilaton. In particular, we have also considered the dilaton field in the background of closed string operators of higher ghost cohomologies:

$$
W_{5} \sim \int d^{2} z e^{-4 \phi-\bar{\phi}} \partial X_{\left(m_{1}\right.} \ldots \partial X_{\left.m_{3}\right)} \bar{\psi}_{m_{6}} e^{i k^{\perp} X} G_{m_{1} \ldots m_{5} m_{6}}
$$

where the G-tensor is symmetric and traceless in $m_{1}, \ldots m_{5}$ (round brackets imply the symmetrization in space-time indices) and $k^{\perp}$ is transversely to $m_{1}, \ldots m_{6}$ directions and

$$
U_{5} \sim \int d^{2} z e^{-4 \phi-\bar{\phi}} \partial X_{\left(m_{1}\right.} \ldots \partial X_{\left.m_{4}\right)} \psi_{m_{5}} \psi_{m_{6}} \bar{\psi}_{m_{7}} e^{i k^{\perp} X} G_{m_{1} \ldots m_{7}}
$$

We have found that, even though the OPE details are quite different in each case, nevertheless in the end one always gets the beta-function equations in the form (22). The crucial point is that the $\sigma$ factor, reflecting the stochasticity of the beta-function, appears to be universal and its value is independent on details of the ghost-matter mixing. Namely, we have found [8] $\sigma=1,541 \ldots$ for the $W_{5}$ insertion and $\sigma=1,538 \ldots$ for the of $U_{5}$ case . In other words, the form of these equations is determined by the corresponding coefficient of the OPE of two operators before the dilaton, and the numerical factor of $\sigma$ What is quite remarkable, the coefficient $\sigma=1,534 \ldots$ before the stochastic term is always the same and 
seems to be invariant on details of the ghost-matter mixing (in the absence of the ghostmatter mixing, of course $\sigma=0$ ). What is even more remarkable, can easily check that in fact

$$
\sigma=\ln \delta
$$

where $\delta=4,669 \ldots$ is the famous Feigenbaum universality constant describing the universal scaling of the iteration parameter in a huge variety of dynamical systems under bifurcations and transitions from order to chaos [7]. Usually this constant can be obtained numerically for the dynamical systems under the bifurcations. In our approach, however, the log of the Feigenbaum constant has emerged analytically, as the limit of convergent series (20) in the stochastic term in the beta-function for various ghost-matter mixing backgrounds. In the next section we will show that the appearance of the Feigenbaum constant in the betafunction for the dilaton in ghost-matter mixing backgrounds is not at all occasional, but is deeply related to the peculiarities of the non-Markovian stochastic processes, associated with the Langevin equations and their implications for the space-time geometry.

\section{Feigenbaum universality and fixed points of stochas- tic RG equations}

To understand the physical meaning behind the appearance of the Feigenbaum constant in (20), it is necessary to analyze the non-Markovian Fokker-Planck (FP) equation describing the stochastic process which can be straightforwardly derived from the Langevin equation (22). We shall present here FP equation for scaling functions $\lambda(q)=\lambda_{0} / q^{4}$

$$
\begin{array}{r}
\frac{\partial P_{F P}(\varphi, \tau)}{\partial \tau}=-\int d^{4} p \int d^{4} q \frac{\delta}{\delta \varphi(p, \tau)}\left(\frac{\delta S_{\varphi}}{\delta \varphi(q, \tau)} P_{F P}(\varphi, \tau)\right) \\
+\sigma^{2} \lambda_{0}^{6} \int d^{4} k_{1} \int d^{4} k_{2} \int \frac{d^{4} p}{p^{4}} \int \frac{d^{4} q}{q^{4}} \int d \xi \\
\alpha_{[-3 \mid 1]} C_{[-3 \mid-3]}\left(\frac{k_{1}+p}{2}\right) \alpha_{[-3 \mid 1]} C_{[-3 \mid-3]}\left(\frac{k_{2}+q}{2}\right) \frac{\delta}{\delta \varphi(p, \tau)} G_{5}(\xi, \tau) \frac{\delta}{\delta \varphi(q, \xi)} P_{F P}(\varphi, \tau)
\end{array}
$$

where $\tau=\log \Lambda$ now plays the role of the stochastic time variable. The Green's function $G_{5}(\xi, \tau, p, q)$ is defined by the cutoff dependence of the two-point correlator of the $V_{5^{-}}$ vertices:

$G_{5}(\xi, \tau)=\int_{\Lambda_{1}} d^{2} z \int_{\Lambda_{2}} d^{2} w|z-w|^{-4} \delta(p+q)=\left(\frac{1+e^{\xi-\tau}}{1-e^{\xi-\tau}}\right)^{2} \delta(p+q), \xi=\log \Lambda_{1}, \tau=\log \Lambda_{2}(27)$ 
We shall look for the anzats solving this equation in the form (for more details see [3] and references therein):

$$
P_{F P}(\varphi, \tau)=\exp \left[-H_{A D M}(\varphi, \tau)\right]=\exp \left[-\int d^{4} p\left\{g(\tau)\left(\partial_{\tau} \varphi\right)^{2}+f(\tau) p^{2} \varphi^{2}\right\}\right]
$$

Substituting it into (26) we find that (28) solves the Fokker-Planck equation provided that the functions $f(\tau)$ and $g(\tau)$ satisfy the following differential equations:

$$
\begin{array}{r}
g^{\prime}(\tau)+4 g(\tau)+\frac{\sigma^{2} \lambda_{0}^{6}}{2}=0 \\
\frac{1}{4} f^{\prime \prime}+\left(1+\frac{1}{4 \tau}\right) f^{\prime}+\left(1+\frac{1}{4 \tau}+\frac{1}{4 \sigma^{2} \lambda_{0}^{6}}\left(1-\frac{1}{\tau^{2}}\right)\right) f-\left(1-\frac{1}{\tau^{2}}\right)\left(e^{-2 \tau}+\frac{1}{4 \sigma^{2} \lambda_{0}^{6}}\right)=1
\end{array}
$$

The first equation is elementary, its solution is given by

$$
g(\tau)=\frac{\sigma^{2} \lambda_{0}^{6}}{2}\left(e^{-4 \tau}-1\right), \quad \tau<0
$$

The second equation on $f(\tau)$ can be reduced to the Bessel type equation by substituting

$$
f(\tau)=\rho(\tau) e^{-2 \tau}+\frac{1}{\sigma^{2} \lambda_{0}^{6}}
$$

The solution is given by

$$
f(\tau)=1+\sigma^{2} \lambda_{0}^{6} e^{-2 \tau}\left(1+J_{\frac{1}{\sigma \lambda_{0}^{3}}}\left(\frac{\tau}{\sigma \lambda_{0}^{3}}\right)\right)
$$

where $J_{\frac{1}{\sigma \lambda_{0}^{3}}}\left(\frac{\tau}{\sigma \lambda_{0}^{3}}\right)$ is the Bessel's function. In terms of the $\tau$ coordinate, the stochastic process, describing the RG flow in ghost-matter mixing backgrounds, evolves in the direction of $\tau=-\infty$. Next, let us study the behaviour of the Fokker-Planck distribution (28), (30), (31) in the conformal limit of $\tau \rightarrow-\infty$ In this limit the exponents become very large and moreover

$$
J_{\frac{1}{\sigma \lambda_{0}^{3}}}\left(\frac{\tau}{\sigma \lambda_{0}^{3}}\right) \sim O\left(\frac{1}{\sqrt{\tau}}\right)<<1
$$

and after rescaling the distribution reduces to

$$
H(\varphi, \tau)=R^{2} \int d^{4} p\left\{e^{-4 \tau}\left(\partial_{\tau} \varphi\right)^{2}+p^{2} e^{-2 \tau} \varphi^{2}\right\}
$$


which is just the ADM Hamiltonian for the $A d S_{5}$ gravity in the temporal gauge [9]. It is easy to see that the $\lambda_{0}^{6}$ parameter has the meaning of the square of the radius $R^{2}$ of the metric.

Let us now analyze in more details the solution (28), (30), (31) of the non-Markovian FP equation, leading to the new space geometry. Let us note first of all that the limit $\lambda_{0} \rightarrow 0$ is not the same as $\lambda_{0}=0$ (ghost-matter mixing absent). The RG flow described by the effective metric (30),(31) must be single-valued; since Bessel's functions at zero argument are single-valued for the integer orders only, this leads to the quantization condition:

$$
\left(\sigma \lambda_{0}^{3}\right)^{-1}=N
$$

Moreover, since $J_{\nu}(\tau) \sim \tau^{\nu}$ as $\tau \rightarrow 0$, the absence of unphysical singularities at $\tau=0$ requires $\mathrm{N}$ to be positive. The quantization condition (32) implies that

$$
\left(\left(\lambda_{0}\right)_{N}\right)^{-3}=N \sigma, \quad e^{\left(\lambda_{0}\right)_{N}^{-3}}=\delta^{N}
$$

implying the iteration law:

$$
\frac{e^{\left(\lambda_{0}\right)_{N+1}^{-3}}-e^{\left(\lambda_{0}\right)_{N}^{-3}}}{e^{\left(\lambda_{0}\right)_{N}^{-3}}-e^{\left(\lambda_{0}\right)_{N-1}^{-3}}}=\delta
$$

with $\delta$ being the Feigenbaum number.

Therefore the Feigenbaum iteration rule (36) determines the scaling of characteristic curvatures of geometries emerging at the fixed points of the stochastic renormalization group. The role of iteration parameter characterizing the bifurcations is played by $\sim e^{-\frac{1}{R^{2}}}$, vanishing at $R=0$ and being finite at large $\mathrm{R}$, as it should be the case for the scaling parameter of the Feigenbaum iteration scheme.

From the quantization condition (34) it is clear that the stochastic renormalization group (22) has fixed points exists for $0<\lambda_{0}<1$, (physically, these points correspond to large curvatures). Moreover the period doublings that lead to the transition to chaos corresponds to $N \rightarrow \infty$, i.e. $\lambda_{0} \rightarrow 0$, which is a singularity. So we reached an amazing conclusion that precisely near singularity our RG flow becomes chaotic. It is tempting to assume that this may be the mechanism which can solve the problem of singularities in string theory. 


\section{Conclusion}

In this Letter we discussed how matter-ghost mixing can radically modify the nature of the world sheet RG flows and lead to the emergence of chaos near curvature singularities. Here we analyzed only dilaton evolution, but the similar picture can be obtained for other massless fields, for example metric [8].

It is amusing that recently chaotic behaviour of metric was discussed in [10] (for earlier papers see [11] and references therein) where the emergence of chaos in supergravity near cosmological singularity was demonstrated in the presence of higher rank antisymmetric tensor fields, i.e. R-R fields. It will be extremely interesting to understand how chaos emerging during cosmological evolution in supergravity can be related to the chaotic nature of RG flows in underlying string theory in the presence of the sources of the background R-R fields.

It is tempting to assume that the resolution of the singularities problem is transition to chaos and emergence of smooth distributions of fields, not restricted on-shell. One can imagine that curvature $R$ is some new "Reynolds" number in string theory and for large $R$ one have transition to chaotic behaviour in a similar fashion like in hydrodynamics there is a transition from a laminar to a turbulent flow. These ideas definitely need further investigation.

I.K. is supported in part by PPARC rolling grant PPA/G/O/1998/00567 and EC TMR grants HPRN-CT-2000-00152 and HRRN-CT-2000-00148. D.P. acknowledges the support of the Academy of Finland under the Project no. 54023 and both I.K. and D.P. acknowledge interesting discussions with T. Damour and the hospitality of Institute des Hautes Etudes Scientifiques (IHES) in Bures-sur-Yvette where part of this work has been done.

\section{References}

[1] A.M.Polyakov, "Gauge Fields and Strings", Harwood Academic Publishers, (1987).

M.B.Green, J.H.Schwarz and E.Witten, "Superstring Theory", vol 1,2 (CUP), (1987).

J. Polchinski, "String Theory", vol 1,2 (CUP), (1998);

[2] D.Fiedan , Phys.Rev.Lett. 45, (1980), 1057; Ann.of Phys.(N.Y.), 163, (1985), 318.

E.S.Fradkin and A.A.Tseytlin, Nucl.Phys. B 261, (1985), 1.

C.G.Callan, D.Friedan, E.J.Martinec and M.J.Perry, Nucl.Phys. B 262, (1985), 593. 
C.G.Callan and Z.Gan, Nucl.Phys. B 272, (1986), 647.

A.B.Zamolodchikov, JETP.Lett.43 (1986),730; Sov.J.Nucl.Phys.46(6) (1987), 1090.

[3] D. Polyakov, Class. Quant. Grav. 18 (2001) 1979 [arXiv:hep-th/0005094].

[4] I. I. Kogan and D. Polyakov, Int. J. Mod. Phys. A 16, 2559 (2001) [arXiv:hepth/0012128].

[5] D. Polyakov, Phys. Rev. D 65, 084041 (2002) [arXiv:hep-th/0111227].

[6] I. I. Kogan and D. Polyakov, Int. J. Mod. Phys. A 18 (2003) 1827 [arXiv:hepth/0208036].

[7] M. J. Feigenbaum, J. Statist. Phys. 19 (1978) 25.

[8] I. I. Kogan and D. Polyakov, to be published

[9] J. de Boer, E. Verlinde and H. Verlinde, JHEP 0008 (2000) 003 [arXiv:hepth/9912012].

[10] T. Damour, M. Henneaux, Phys. Rev. Lett. 85 920, (2000) hep-th/0003139;Phys. Rev. Lett. 864749 (2001) hep-th/0012172; Gen. Rel. Grav. 32 (2000) 2339.

T. Damour, M. Henneaux, B. Julia and H. Nicolai, Phys. Lett. B 509 (2001) 323 [arXiv:hep-th/0103094].

T. Damour, M. Henneaux and H. Nicolai, Phys. Rev. Lett. 89 (2002) 221601 [arXiv:hep-th/0207267].

T. Damour, Int. J. Mod. Phys. A 17 (2002) 2655.

[11] V. D. Ivashchuk and V. N. Melnikov, J. Math. Phys. 41 (2000) 6341 [arXiv:hepth/9904077]. 